Mitteilungen der Österreichischen Geographischen Gesellschaft, 158. Jg. (Jahresband), Wien 2016, S. 353-358

\title{
33. InTERnationaler Geographischer Kongress „Shaping Our Harmonious World“ Peking [Beijing], 21.-25. August 2016
}

\author{
Peter JORDAN, Wien* \\ mit 4 Abb. im Text
}

Die Internationale Geographische Union (IGU) hält alle vier Jahre ihre großen Kongresse ab, im Rahmen derer jeweils auch die Generalversammlung dieser globalen Dachorganisation der Geographie mit den Wahlen des Leitungsgremiums und der Bestellung der Kommissionen mit ihren Funktionsträgern stattfindet. Zwischen den großen Kongressen war die IGU in jüngerer Zeit dazu übergegangen, jährlich Regionalkonferenzen zu veranstalten, die den Kongressen in Bezug auf fachliches Angebot, Größenordnung und globaler Herkunft der Teilnehmer aber kaum nachstanden. So gab es seit dem letzten Kongress im Jahr 2012 in Köln Regionalkonferenzen in Kyōto (2013), Krakau [Kraków] (2014) und Moskau [Moskva] (2015).

\section{Teilnahme und Organisation}

Der 33. Kongress in Peking ragte aber nun doch - jedenfalls an der Zahl der Teilnehmer gemessen - deutlich über die Regionalkonferenzen hinaus. Mit 4.299 Teilnehmern (IGU-Newsletter NS Nr. 20, S. 18) schlug er sogar alle bisherigen Rekorde. Natürlich kam ein Großteil (2.349) aus dem Veranstalterland selbst, doch waren immerhin 101 Länder vertreten und kamen viele auch aus Europa und Nordamerika. Länder mit mehr als 30 bzw. 10-30 Teilnehmern zeigen die Abbildungen 1 und 2. Österreich dürfte nur durch eine Person, den Berichterstatter, vertreten gewesen sein.

Der Kongress war von der Chinesischen Geographischen Gesellschaft und vom Institut für Erdwissenschaften und Naturressourcen an der Chinesischen Akademie der Wissenschaften organisiert worden und fand im größten Kongresshaus Pekings und wohl auch Chinas, dem China National Convention Centre (Abb. 3) im Bereich des Olympischen Parks, statt. Der Kongress bot neben einem Fachprogramm mit 262 Sitzungen in zahlreichen parallelen Strängen, neun Plenarvorträgen und etlichen sogenannten ,public lectures“ im Sinne von längeren Vorträgen außerhalb des Sitzungsschemas zu aktuellen Themen, gesellige Ereignisse wie den Begrüßungsempfang und das Galadiner, eine Fachausstellung sowie zahlreiche Exkursionen während des Kongresses sowie im Anschluss an ihn - so z.B. nach Lhasa in Tibet oder Urumqi in Singkiang.

\footnotetext{
* Hofrat Prof. h.c. Univ.-Doz. Dr. Peter JoRdan, Institut für Stadt- und Regionalforschung, Österreichische Akademie der Wissenschaften, Postgasse 7/4/2, A-1010 Wien; E-Mail: peter.jordan@oeaw.ac.at, http://www. oeaw.ac.atisr
} 


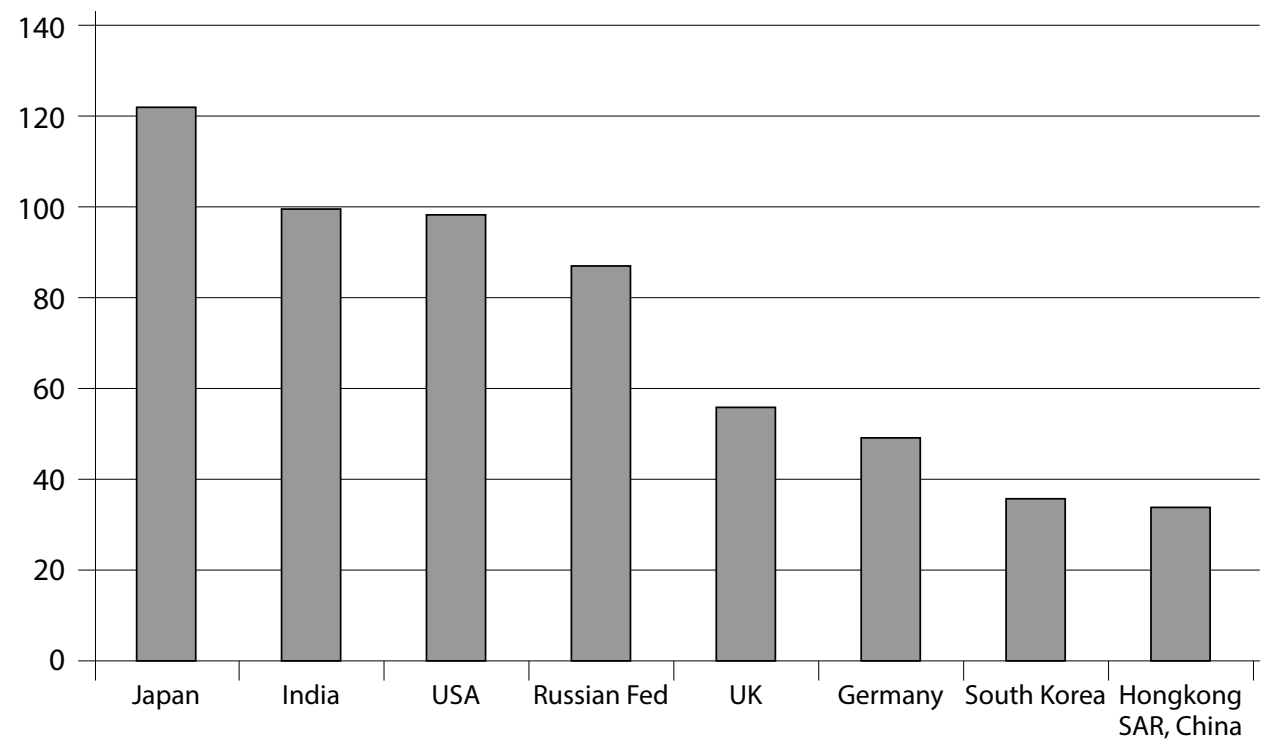

Quelle: IGU-Newsletter NS Nr. 20, S. 19

Abb. 1: Länder mit mehr als 30 Teilnehmern (außer Festland-China)

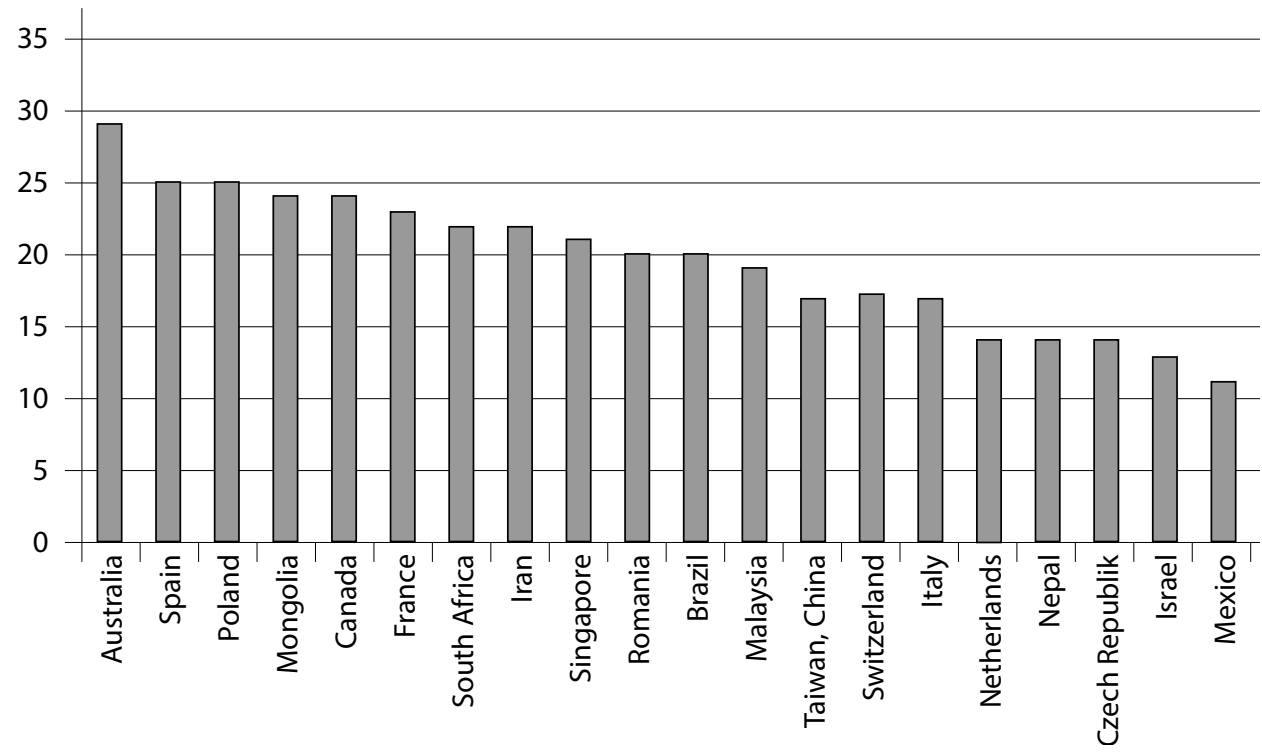

Quelle: IGU-Newsletter NS Nr. 20, S. 19

Abb. 2: Länder mit 10-30 Teilnehmern 


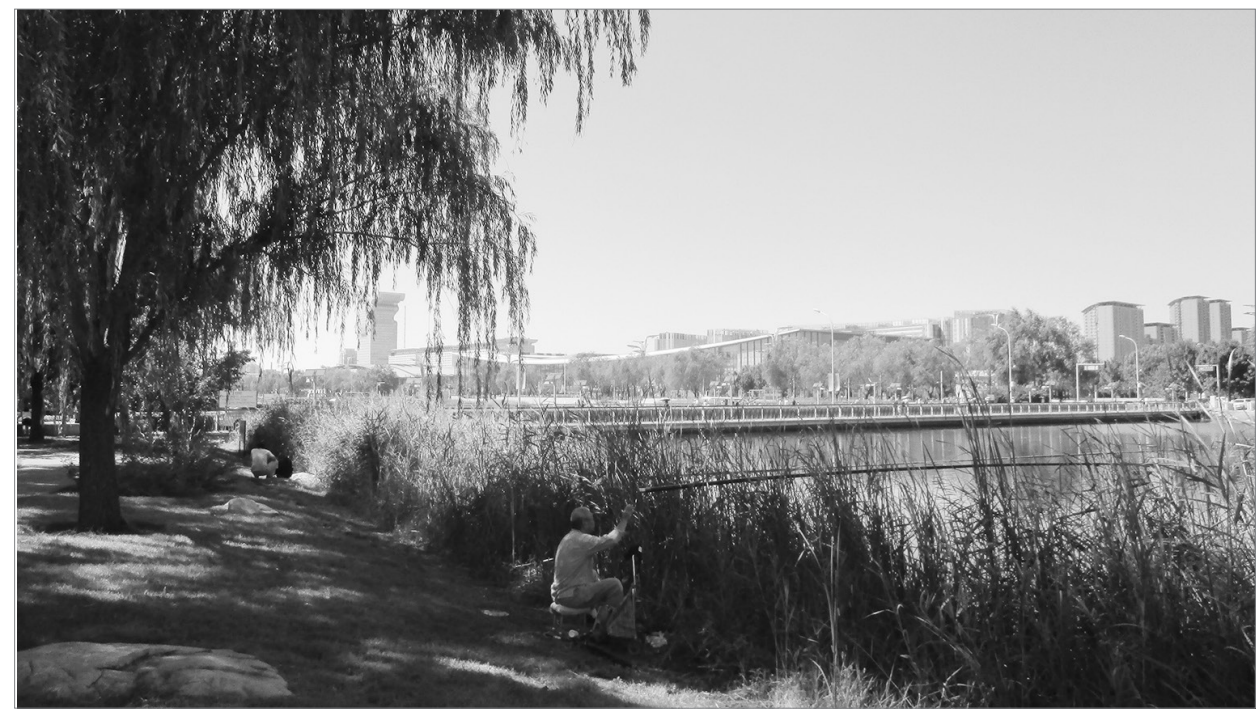

Foto: JORDAN 2016

Abb. 3: Im Bildhintergrund der Tagungsort, das China National Convention Centre

\section{Fachliche Inhalte}

Neben der bemerkenswert nüchternen Eröffnung mit einigen programmatischen Reden markierten vor allem die über das Tagungsprogramm verteilten Plenarvorträge (keynotes) die Entwicklungsrichtungen der Geographie oder führten an die Geographie des Gastlandes heran. Mark STAFFORD Sмітн (Australien) sprach über „Geographical Sciences and Future Earth: Research for Solutions, from Local to Global Scales“, Bojie Fu (China) über „Understanding Chinese Geography: Linking Science and Society“, Woo-ik Yu (Republik Korea) über „Back to the Future: Reasoning the Territorial Way of Life“", das Mitglied unseres Herausgeberkomitees Denise PuMAIN (Frankreich) über „Urban Dynamics and Geodiversity: From Theory to Modeling“, Mark W. Rosenberg (Kanada) über „A $21^{\text {st }}$ Century Agenda to Shape ,Our Harmonious Worlds' through Research on the Geographies of Health and Care“, Dahe QIN (China) über „Climate Change and Its Impact“, Michael Meadows (Südafrika) über „Geography and Future Earth: Perspectives from Africa“ und Benno WeRLEN (Deutschland) über "Global Understanding for Global Sustainability“. Virginie MamadouH (Niederlande) und Takashi YAMAZAKI (Japan) schließlich referierten zum Thema „From Inter-state to Multiscalar Political Geographies“.

Unter den „public lectures“ soll der Vortrag von Anthony YeH (Hongkong [Xianggang]) hervorgehoben werden, weil er ein Schlaglicht auf eine durchaus dramatische aktuelle Entwicklung im Gastgeberland warf: auf den rasanten Verstädterungsprozess, das Abheben der Metropolen und die beginnende Entvölkerung des ländlichen Raumes. YEH, der die staatliche chinesische Politik sehr kritisch beurteilte (Als Bürger Hongkongs ist das offenbar möglich.), teilte die Entwicklung des Städtesystems und des Stadt-Land-Verhältnisses in China nach dem Zweiten Weltkrieg in vier Perioden: (1) in die Periode von 1949 bis 1977, als die offizielle Politik die Wanderung behinderte und es dadurch zu einem relativen Anstieg der ländlichen Bevölkerung kam; (2) in die Periode von 1978 
bis 1987, als eine Politik der polyzentrischen Entwicklung und einer Industrialisierung auch des ländlichen Raums betrieben wurde und dadurch zwar eine Urbanisierung im soziologischen Sinn erfolgte, das Zentrensystem aber sehr ausgewogen blieb; (3) in die Periode von 1988 bis 2000, als die Landreform des Jahres 1988 einen Bodenmarkt geschaffen und Kapital für Investitionen in die städtische Infrastruktur generiert hatte, wodurch es zu einem Urbanisierungsschub kam; (4) in die Periode ab 2001, als sich der tertiäre Sektor der Wirtschaft mächtig entwickelte, das Bruttoinlandsprodukt um durchschnittlich 3,8\% pro Jahr anstieg und das die Urbanisierung enorm beschleunigte. In dieser letzten Periode kam es also zu einer nachholenden Entwicklung gegenüber dem politischen Westen, und sie ließ die Metropolen mit ihren Central Business Districts (CBDs) emporschießen. Die Rangliste der Großstädte nach ihrer Einwohnerzahl dehnte sich, und besonders die größten Metropolen Peking, Shanghai sowie die Agglomeration an der Mündung des Perlflusses [Zhū Jiāng] mit Kanton [Guangzhou], Shenzhen und Hongkong hoben ab. Modellrechnungen unter der Annahme, dass die Voraussetzungen und auch die Politik sich nicht ändern, sagen Peking für das Jahr 2030 eine Einwohnerzahl von 38 Millionen (2015: 20 Mio.) und Shanghai eine von 28 Millionen (2015: 24 Mio.) voraus. Erstmals würden in China auch Dritte-Welt-Phänomene wie Slums in Erscheinung treten. Der geplante Vollausbau des Hochgeschwindigkeitsnetzes der Bahn bedeutet Anthony YEH zufolge 25\% mehr Wachstum für die größten Städte und 25 statt 23 Städte mit mehr als 10 Millionen Einwohnern.

Im Namen der Joint IGU/ICA Commission on Toponymy hatte der Berichterstatter als deren Stellvertretender Vorsitzender eine Sitzung zum Thema „Place Names as Social Constructs“ organisiert, in deren Rahmen schließlich 17 Vorträge gehalten wurden. Peter JoRDAN selbst leitete gemeinsam mit Cosimo Palagiano (Italien), dem IGU-Vorsitzenden der Kommission, die Sitzung und steuerte den Vortrag ,The Ups and Downs of German Exonym Use. Use of Exonyms as an Indicator of the User Community's Prestige" bei.

Die aktivsten der insgesamt 41 IGU-Kommissionen waren die Commission on Geography of Tourism, Leisure and Global Change (Vorsitz: Dieter MüLlER, Deutschland) mit 16 Sitzungen, die Commission on Geographical Education (Vorsitz Clare Brooks, Vereinigtes Königreich) mit 11 Sitzungen, die Urban Commission: Urban Challenges in a Complex World (Vorsitz: Celine RozenBLAT, Schweiz) mit 11 Sitzungen und die Commission on Political Geography (Vorsitz: Virginie Mamadoun, Niederlande, und Alexander Murphy, USA) mit 8 Sitzungen.

Breiten Raum nahm auch das von Benno WeRLEN (Deutschland) bestens organisierte und propagierte International Year of Global Understanding ein, das die Notwendigkeit eines umweltverträglichen und nachhaltigen Wirtschaftens noch stärker ins Bewusstsein zu rufen versucht.

\section{Generalversammlung}

An der Generalversammlung der IGU, die in drei Teilen durchgeführt wurde, nahmen die Vertreter von 46 der insgesamt 64 IGU-Mitgliedsländer teil. Österreich war, delegiert vom österreichischen IGU-Komitee, durch den Berichterstatter vertreten. Die Generalversammlung wählte nach dem Ende der Amtsperiode von Vladimir Kolossov (Russland) einen neuen Präsidenten und die übrigen Mitglieder des neuen Exekutivkomitees, bestätigte die bestehenden bzw. bestellte neue Kommissionen und deren Funktionäre und entschied sich für den Tagungsort des übernächsten IGU-Kongresses im Jahr 2024. (Der des nächsten im Jahr 2020 steht mit Istanbul [İstanbul] bereits fest.)

Zum neuen Präsidenten für die Amtsperiode 2016-2018 wurde der Japaner Yukio HiмiYAмA gewählt. Das Exekutivkomitee besteht nun für dieselbe Amtsperiode aus den folgenden weiteren Mitgliedern: Michael MeAdows (Generalsekretär, Südafrika), Vladimir Kolossov (Past President, 
Russland), Joos Droogleever FortuiJn (Erste Vizepräsidentin, Niederlande), Elena DELL'AgNeSE (Vizepräsidentin, Italien), Iain HAY (Vizepräsident, Australien), Zhou CHENGHU (Vizepräsident, China), R.B. Singh (Vizepräsident, Indien), Barbaros GöNEnçGIL (Vizepräsident, Türkei), Nathalie Lemarchand (Vizepräsidentin, Frankreich) und Rémy Tremblay (Vizepräsident, Kanada).

Unter den Kommissionen wurde auch die Joint IGU/ICA Commission on Toponymy mit ihrer bisherigen Leitung (also dem Berichterstatter als Stellvertretenden Vorsitzenden) bestätigt.

Unter den vier Bewerbungen um den übernächsten IGU-Kongress im Jahr 2024 (Dublin/Baila Átha Cliath), Irland; Melbourne, Australien; Kopenhagen [København], Dänemark - Malmö, Schweden; Prag [Praha], Tschechien) entfielen die meisten Stimmen auf Dublin/Baila Átha Cliath.

\section{Ehrungen und kommende IGU-Veranstaltungen}

Der Kongress schloss mit einer Reihe von Ehrungen. Den IGU Commission Excellent Award 2015 für die beste Leistung einer Kommission erhielt die Commission on Geographical Education (Vorsitz Clare Brooks, Vereinigtes Königreich), die IGU Planet and Humanity Medal Carl FoLKE (Schweden). Die IGU Lauréats d'Honneur gingen an Ian BurTon (Kanada), Maria Dolores GARCIAS-RAMON (Spanien) und Benno WeRLEN (Deutschland).

Schließlich vermittelten die Vertreter der nächsten IGU-Regionalkonferenz im Jahr 2018 in Québec (Kanada) und des nächsten IGU-Kongresses im Jahr 2020 in Istanbul (Türkei) einen Vorgeschmack auf diese Ereignisse. Die nächste weltweite IGU-Tagung wird aber mit der Thematic Conference „Geographies for Peace“ in La Paz (Bolivien) schon vom 23. bis 25. April 2017 stattfinden.

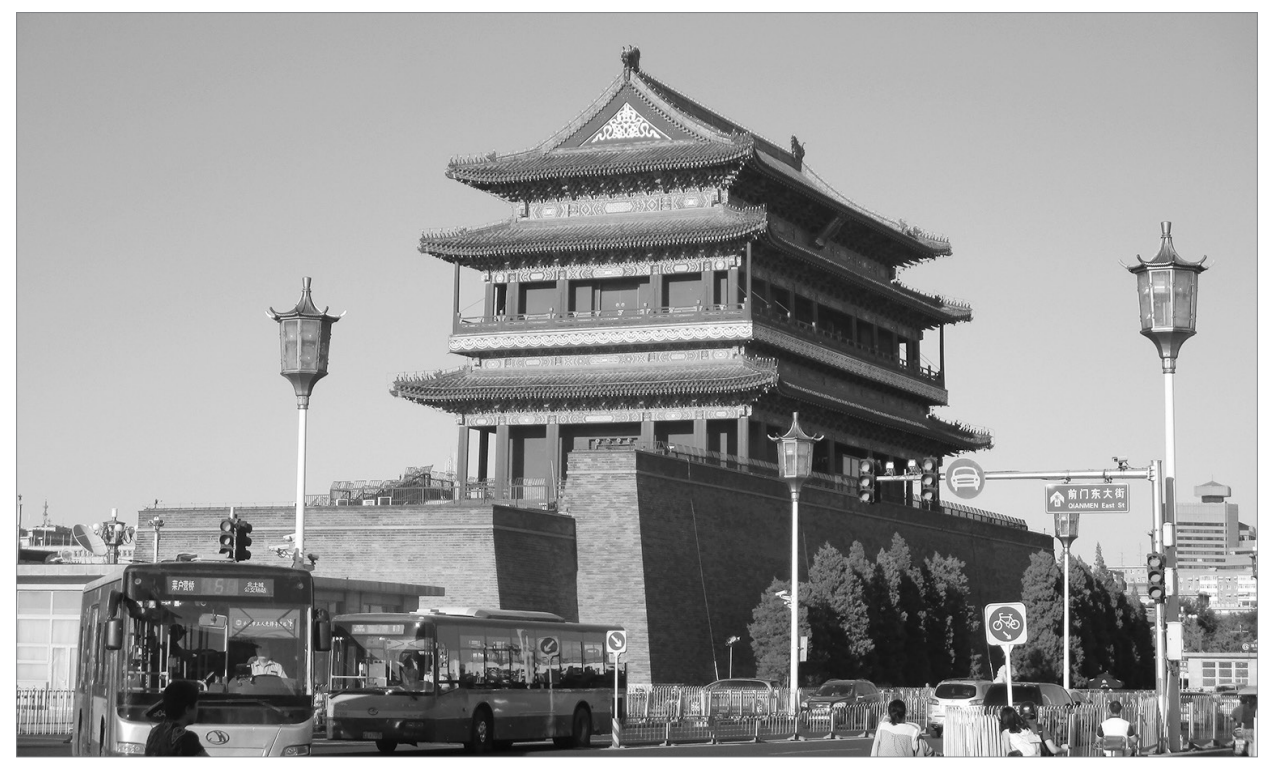

Foto: JORDAN 2016

Abb. 4: Südlich des Platzes des Himmlischen Friedens [Tiān`ānmén Guăngchăng] 


\section{Ein österreichisches Resümee}

Der IGU-Kongress war gut organisiert und reich an Inhalten. Auch die Stadt Peking (Abb. 4) und das Gastland boten genügend Anreize, auch die weite Reise aus Österreich auf sich zu nehmen. Dennoch war die österreichische Geographie kaum präsent. Das muss auch schon für die IGU-Veranstaltungen seit dem IGU-Kongress in Köln festgestellt werden und gilt nicht nur für das Fachliche, sondern auch für den organisatorischen Bereich, also die Leitungsebenen der IGU.

Dieses geringe Engagement steht in auffälligem Gegensatz zu jenem in der weltweiten Dachorganisation der Kartographie, der Internationalen Kartographischen Vereinigung (International Cartographic Association, ICA), in der Österreich eine seiner Größe mehr als proportionale Rolle spielt, von Georg GARTNER als Präsidenten eine Amtsperiode lang hervorragend vertreten wurde und auch immer wieder Commission Chairs stellt. Es steht auch im Gegensatz zu nicht so lang zurückliegenden Zeiten in der IGU.

Das aktuelle Manko lässt sich günstigstenfalls mit der vorzugsweisen Ausrichtung der österreichischen Geographie auf den deutschen Sprachraum erklären, wodurch vielleicht alle freien Kräfte und Mittel absorbiert werden. Wissenschaft ist aber dem Prinzip nach international und muss sich der globalen Konkurrenz und Kritik stellen. So selten wie wir Ergebnisse der französischen, spanischen, italienischen oder ungarischen Geographie rezipieren, wenn die nicht auf der globalen Bühne präsentiert werden, so wenig wird unsere Arbeit international sichtbar und angemessen gewürdigt, wenn sich ihre Präsentation auf den deutschen Sprachraum beschränkt. 